\title{
Detailed Model for Hot-Dense Aluminum Plasmas Generated by an X-ray Free Electron Laser
}

O. Ciricosta, ${ }^{1, \text { a) }}$ S. M. Vinko, ${ }^{1}$ H.-K. Chung, ${ }^{2}$ C. Jackson, ${ }^{1}$ R.W. Lee, ${ }^{3}$ T. R. Preston, ${ }^{1}$ D. S. Rackstraw, ${ }^{1}$ and J. S. Wark ${ }^{1}$

1) Department of Physics, Clarendon Laboratory, University of Oxford, Parks Road, Oxford OX1 3PU, $U K$

${ }^{2)}$ Atomic and Molecular Data Unit, Nuclear Data Section, IAEA, P.O. Box 100, A-1400, Vienna, Austria

3) Department of Physics, University of California, Berkeley, California 94720, USA

(Dated: 9 February 2016)

The possibility of creating hot-dense plasma samples by isochoric heating of solid targets with high-intensity femtosecond X-ray lasers has opened up new opportunities in the experimental study of such systems. A study of the X-ray spectra emitted from solid density plasmas has provided significant insight into the X-ray absorption mechanisms, subsequent target heating, and the conditions of temperature, electron density, and ionization stages produced (Vinko et al. Nature 482, 5962). Furthermore, detailed analysis of the spectra has provided new information on the degree of ionization potential depression in these strongly coupled plasmas (Ciricosta et al. PRL 109, 065002). Excellent agreement between experimental and simulated spectra has been obtained, but a full outline of the procedure by which this has been achieved has yet to be documented. We present here the details and approximations concerning the modelling of the experiment described in the above referenced work. We show that it is crucial to take into account the spatial and temporal gradients in simulating the overall emission spectra, and discuss how aspects of the model used affect the interpretation of the data in terms of charge-resolved measurements of the ionization potential depression.

PACS numbers: 52.20.-j, 52.65.-y, 52.25.Os, 52.70.La

\section{INTRODUCTION}

The first experiment where the high intensity X-ray pulse of the Linac Coherent Light Source (LCLS) interacts with a solid target $^{1,2}$ - a thin Al foil - has proved the feasibility of generating solid-density samples of hotdense matter, at temperatures up to $\sim 200 \mathrm{eV}$ and in well known conditions, by using an X-ray free-electron laser (FEL). This result offers a new platform for the investigation of the basic properties of these strongly coupled systems - of primary relevance to planetary ${ }^{3}$, stellar $^{4,5}$ and fusion science ${ }^{6,7}$ - and for a much needed validation of the models currently used to predict their behaviour. Indeed the same experiment has already allowed for the first charge-resolved measurement of continuum lowering in hot-dense matter ${ }^{8}$, and for the measurement of femtosecond collisional ionization rates under the same conditions $^{9}$, the results of which both challenge the standard models used in dense-plasma simulations.

Although the experiment and its results have been discussed in the corresponding publications (as listed above), a detailed description of the simulation work that underlies the interpretation of the data, and of the corresponding approximations, is not yet available in the literature. This paper aims to address this deficiency. The large degree of IPD that has been inferred from the experimental spectra has led to a renewed interest

a) Electronic mail: orlando.ciricosta@physics.ox.ac.uk in this phenomenon ${ }^{10-15}$, and we use this opportunity to address several comments that have been made about the interpretation of the experimental data, which is strongly constrained by the predictive nature of the simulations.

\section{A. The experiment}

We briefly recall here the general experimental technique and its main results, referring to the experimental paper $^{1}$ for a detailed description of the setup.

In the experiment, the X-ray pulse of the LCLS ( $\sim 1 \mathrm{~mJ}, 80 \mathrm{fs}$ ) was focused on a $1 \mu \mathrm{m} \mathrm{Al}$ foil lying at $45^{\circ}$ to the incident beam, at peak intensities exceeding $10^{17}$ $\mathrm{W} \mathrm{cm}^{-2}$, whilst varying the photon energy of the FEL in the range between 1460 and $1830 \mathrm{eV}$. The plasma was then diagnosed by $\mathrm{K}_{\alpha}$ spectroscopy, using a flat-crystal spectrometer sensitive to the $\mathrm{X}$-ray emission in the range $1460-1680 \mathrm{eV}$. The experimental spectra ${ }^{1,2}$ recorded as a function of the FEL photon energy are shown in Fig. 1: the observed peaks correspond to $\mathrm{K}_{\alpha}$ emission lines from ions with a different number of L-shell holes ( $K_{\alpha}$ satellites), and having either one (lines at energies below 1600 $\mathrm{eV}$ ) or two (above $1600 \mathrm{eV}$ ) K-shell holes in the initial state of the corresponding radiative transition. The roman numeral associated to each of these lines corresponds to the total number of ionized K- and L-shell electrons from the emitting ions.

As the FEL photon energy is progressively increased, a varying number of spectral lines is observed. First, when 

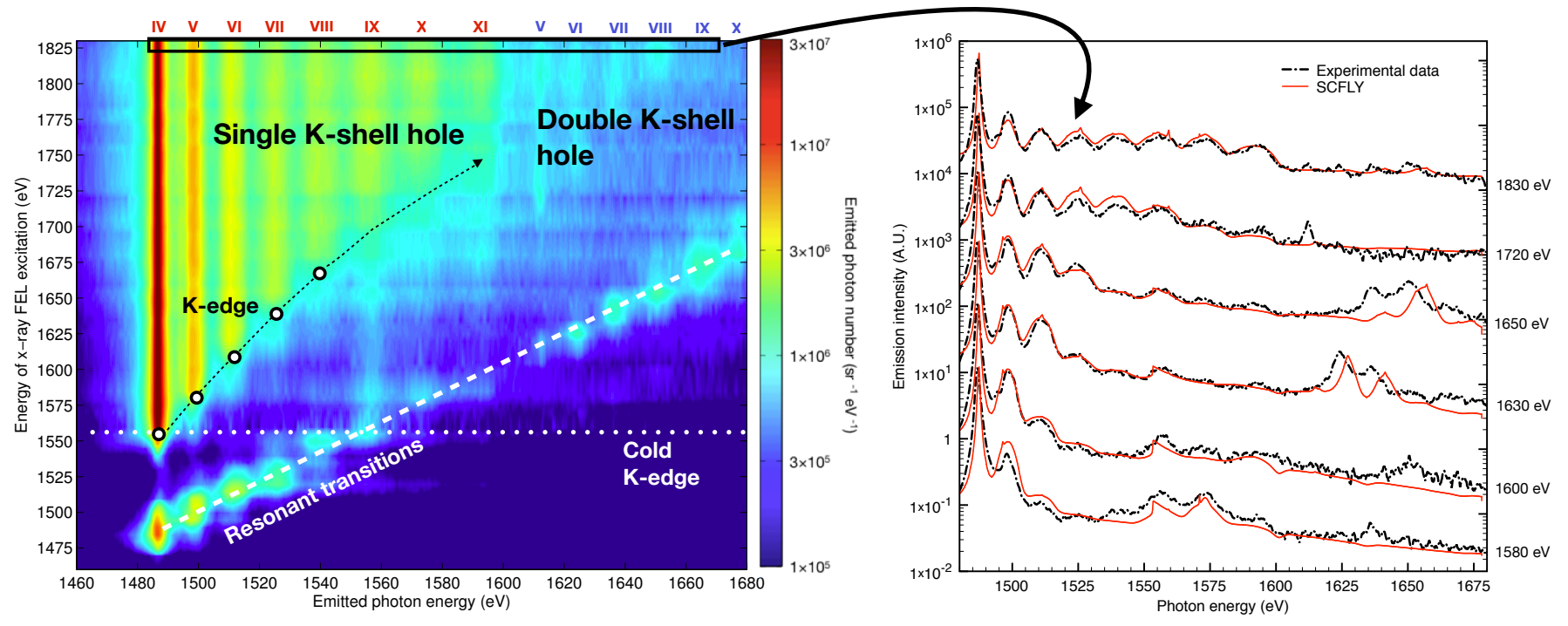

FIG. 1. (Color online) Experimental $K_{\alpha}$ spectra plotted as a function of the FEL photon energy (left). The spectra are compared with SCFLY simulations for a few FEL photon energies (right). The curves are displaced in intensity for clarity, and the simulations are corrected by an artificial bremsstrahlung background, at the maximum temperature predicted by the simulations.

the photon energy matches the energy of a K- to L-shell transition, the corresponding L- to K-shell emission line is observed as a resonance ${ }^{1,2,16}$; second, except for these easily identifiable resonances, the spectra exhibit significant line emission only for those specific ions for which the photon energy exceeds the ionization threshold for a $\mathrm{K}$-shell electron ${ }^{1,8}$. This second observation yields ionization thresholds which are inconsistent with the prediction of the standard model of continuum lowering, the Stewart and Pyatt model ${ }^{17}$, but are still described by another analytical model, the Ecker and Kröll model ${ }^{18}$ (both models are described in section IC).

Interestingly, both models were later found to be inconsistent with the disappearance of the $\mathrm{He}_{\beta}$ and $\mathrm{Ly}_{\beta}$ emission lines, as a function of the ion density, from hotter laser-compressed $\mathrm{Al}$ samples generated in optical-laser experiments at the Orion facility ${ }^{10,19}$, providing further motivation that validation of any of the models used in these high-density plasma conditions is sorely needed.

Also shown in Fig. 1 is a comparison between the experimental spectra and our simulations, for spectra recorded at a few specific FEL photon energies. The simulations represent space- and time-integrated spectra calculated with the SCFLY code (using the Ecker and Kröll model for the continuum lowering), which will be presently described in more detail. It is worth noting that, in spite of the excellent agreement with the data, such calculations are not a result of a fit, but are rather a prediction purely based on the measured FEL parameters. The only user corrections in Fig. 1 are the introduction of a bremsstrahlung background, which takes into account the extremely low-level broad-band emission that lasts far longer than the simulation timescale, and whose only result is to fill in the zones of the spectra with no line emission, and an estimated broadening of the $\mathrm{He}_{\alpha}$ lines $(\sim 5 \mathrm{eV})$, taking into account broadening effects, not included in the code nor easily estimated in the sort of plasma conditions described here. We will describe the details of such simulations throughout the paper. It should also be noted that the two high-energy features observed at the two lowest FEL photon energies, and not reproduced by the simulations, are consistent with a two-photon absorption process where two electrons are excited at the same time from the $\mathrm{K}$-shell to the L-shell, a higher order process neglected by the code.

\section{B. The SCFLY model}

In order to model the experimental results, we performed atomic-kinetics simulations using the collisionalradiative SCFLY code. Since the full capabilities of the code itself have not been described in a dedicated paper, and this has generated some misunderstanding about the accuracy of its results (e.g. the code is described as screened hydrogenic in a recent paper ${ }^{15}$ ), it is worth briefly summarizing here its main features.

SCFLY is a thoroughly revised version of the widelyavailable FLYCHK suite ${ }^{20,21}$, specifically tailored for Xray laser problems. The main upgrades consist in a self consistent temperature calculation - derived by the energy balance between the absorbed and emitted radiation and the variation of internal energy of the ions in the system - and in a larger extent of super-configurations ${ }^{22}$ used to describe the atomic physics of the system. Another improvement is the treatment of the off-resonant radiative pumping of bound-bound transitions. Importantly, the atomic model is modified from the simple 
screened hydrogenic model used in FLYCHK, and rather uses calculated atomic quantities that are given as an input by the user, which in the $\mathrm{Al}$ case $\left(\mathrm{see}^{1}\right)$ were calculated by the DHS code ${ }^{23}$. A super-configuration treatment, defining super-shells by principal quantum number, is employed to produce population distributions by solving the non-LTE (local thermal equilibrium) rate equations. A more detailed atomic model using relativistic configurations calculated by the DHS code was used for spectral synthesis where energy levels, statistical weights and radiative data are applied to produce frequency-dependent emissitivities and opacities. Population distributions of relativistic configurations belonging to a super-configuration (or a super-shell) were determined by a Boltzmann statistics as a function of electron temperature. As a single transition between two superconfigurations consists of multiple transitions of relativistic configurations belonging to each super-configuration, the resulting spectra of SCFLY model produces supertransition arrays consisting of UTA (unresolved transition arrays $)^{20}$.

We stress that for the $\mathrm{Al}$ work all of the possible superconfigurations obtained by redistributing the number of electrons in each ionic stage between the K-, L- and M-shell have been taken into account ${ }^{1}$. This is in contrast to the usual practice of restricting the number of configurations and/or ion stages in order to reduce the duration of a calculation, but clearly allows us to predict the plasma evolution without any user bias on the final plasma conditions; note that $\mathrm{N}$ and higher shells need not be included as they would be excluded from the calculations in any case due to pressure ionization, using any of the continuum lowering models available.

The opacity effects on the populations are taken into account in the code using an escape factor formalism ${ }^{24}$, however, it has been shown that the emission from the target itself has negligible effect on the populations, and they are completely dominated by the intensity of the FEL radiation and highly collisional nature of the system $^{16}$. In the transverse direction, given that the evolution of the populations at a given point in the plasma will be a function of the laser intensity experienced locally, we weight and integrate different simulations with the appropriate peak intensity (see section IIB), using the spatial profile measured during the experiment by imprints ${ }^{25}$ on $\mathrm{PbWO}_{4}$. Although the self-emission does not impact on the populations, the resultant spectra are, at the peak of the K-shell lines, optically thick, and in calculating the spectra the standard equation of radiation transfer is used to predict the emission using the frequency-dependent emissivities and opacities generated by SCFLY.

The X-ray laser radiation field used in the calculations is assumed to be Gaussian both in time, with a full width at half maximum (FWHM) of $80 \mathrm{fs}$, and in frequency, with a fractional bandwidth of $0.5 \%$. For the time-dependent calculations shown later on in this paper, the peak of the pulse occurs at 80 fs. Although the LCLS is a SASE source (Self-Amplified Spontanous Emission), characterized by a chaotic spiked temporal structure $^{26}$, the choice of a Gaussian envelope is justified by the comparison with simulations performed using a spiked temporal profile with the same fluence and duration, showing a negligible effect of the time profile on the final results, as discussed in section IID. The SASE chaoticity may however explain the poor reproducibility of the line intensity for the double core-hole lines shown in Fig. 1 (lines at photon energies above $1600 \mathrm{eV})^{27}$.

The energy deposition process, driven by single-photon absorption events, is intrinsically included in the simulation via the rate equations. Although this is similar to previous FEL work in the non-collisional regime ${ }^{27-29}$, the instantaneous thermalization granted by the high collisionality of the Al plasma (as discussed in section II C) also allows for a complete description of the temperature evolution of the system as it transits from a cold solid to a highly-ionized solid-density plasma. Such a description would be at best extremely challenging for an opticallaser driven solid.

When choosing the total energy delivered onto the target in the simulations, a slightly better agreement between the simulations and experiment was found by using the nominal beamline transmission $(\sim 50 \%$ due to the nominal reflectivities of the optical components) rather than the measured value of $\sim 30 \%^{30}$, which itself is subject to some degree of error. This choice, however, affects mainly the resonant features, sensitive to small variations in peak fluence, while producing a negligible change in the $\mathrm{K}_{\alpha}$ lines pumped by photoionization processes.

\section{Continuum lowering models}

In this section we briefly outline the two analytical models that we used in our simulations in order to account for the continuum lowering, or ionization potential depression (IPD), and describe how they are implemented in the calculations.

The standard model used in plasma simulations for the IPD is the 1966 model by Stewart and Pyatt (SP) ${ }^{17}$. According to this model, which intrinsically assumes weak coupling and thermal equilibrium, the ionization threshold to obtain an ion of charge $z$, in a plasma at temperature $T$ and electron density $n_{e}$, is reduced with respect to case of an isolated ion by

$$
\Delta I_{S P}=\frac{3}{2} \frac{z e^{2}}{4 \pi \epsilon_{0} \lambda_{D}} \frac{\left[\left(a / \lambda_{D}\right)^{3}+1\right]^{\frac{2}{3}}-1}{\left(a / \lambda_{D}\right)^{3}},
$$

where $\lambda_{D}$ is the Debye length including ions and electrons, and the length $a$ is defined by $4 \pi a^{3} / 3=z / n_{e}$.

A model which doesn't rely on weak coupling, but still assumes thermal equilibrium, was put forward in 1963 by Ecker and Kröll $(\mathrm{EK})^{18}$. Instead of deriving a single formula for the IPD, as in the SP model, the EK model derives two limiting forms for the IPD, depending 
on whether the total particle density $n=n_{e}+n_{i}$ is above (strong coupling), or below (weak coupling), the critical density defined by:

$$
n_{\text {crit }}=\frac{3}{4 \pi}\left(\frac{4 \pi \epsilon_{0} k_{B} T}{Z^{2} e^{2}}\right)^{3},
$$

where $Z=13$ for $\mathrm{Al}\left(n_{\text {crit }} \simeq 1.3 \times 10^{20} \mathrm{~cm}^{-3}\right.$ at $\left.200 \mathrm{eV}\right)$. For densities below this value the EK model predicts the standard form of the IPD described within the DebyeHückel (DH) theory ${ }^{31}$, the same limiting form that can be retrieved in the SP model, by taking the limit of Eq. 1 for $\lambda_{D} \gg a$. For densities above critical, as is the case in our experiment, the EK model predicts that the ionization energy required to further ionize an ion of charge $(z-1)$ is reduced by

$$
\Delta I_{E K}=C \frac{z e^{2}}{4 \pi \epsilon_{0} r_{\mathrm{EK}}}
$$

where $r_{\mathrm{EK}}$ is defined by $4 \pi r_{\mathrm{EK}}^{3} / 3=1 / n$ ( $n$ being, again, the total particle density), while $C$ is an unknown multiplicative factor, but such that $C \sim 1$ by construction. Ecker and Kröll propose determining this factor by joining the form (3) and the DH formula at the critical density, but they acknowledge that this assumption can't be justified, as the approximation leading to the $\mathrm{DH}$ solution breaks down near the critical density ${ }^{18}$. Aware of this caveat, and based on the experimental results, in our work we chose instead to simply assume $C=1$ and in keeping with the notation used in the previous work ${ }^{8}$ - we will still refer to Eq.3 with $C=1$ as the EK model.

In our simulations, at each time step of the time dependent calculation, the local values of $n_{e}, n_{i}$ (fixed), $T$ and average ionization are used to calculate the instantaneous values of $\lambda_{D}, a$ and $r_{\mathrm{EK}}$; then these values are used to calculate the different instantaneous IPDs for each of the 13 charge states in the system, using Eq. 1 for the SP case, and Eq. 3 with $C=1$ for EK. The IPD values affect the rate equations in two ways. First, they modify all the bound-free transition energies, and thus the absorption edges, according to the very definition of IPD. Second, all the excited ions whose excitation energy is above the IPD-reduced threshold to ionize their outermost electron are instantaneously ionized, and the corresponding superconfigurations are thus removed from the rate equations. This operation corresponds to the physical process of pressure ionization.

\section{PLASMA EVOLUTION}

The excellent agreement between experiment and simulations shown in Fig 1, together with the relative simplicity of the energy deposition process, provide confidence in the ability of the simulations to correctly track the local time evolution of the plasma. This in turn allows us to have a closer look at the microscopic processes occurring in the system, to understand how these processes manifest themselves in the observed space- and time-integrated data, and finally to extract information on the relevant physics from the data.

This section is dedicated to the first two steps of this process and to the impact of the approximations used in the calculations on the final results, whilst section III describes the final step, in the specific case of extracting measurements of continuum lowering from the data.

\section{A. Time dependent simulations and collisions}

In order to understand how the plasma evolution relates to the spectra observed at different FEL photon energies, let us first restrict our attention to a local simulation for the two limiting cases shown in Fig. 1. To this end, Fig. 2 shows the time-dependent spectrum, temperature and charge state distribution (CSD) at the FEL focus, when the system is either pumped at $1830 \mathrm{eV}$ (left), or $1580 \mathrm{eV}$ (right). Much of the physics governing the plasma evolution can be understood by comparing the CSD with the temperature evolution in the two cases.

Firstly we note that the number of charge states available in the plasma is very similar in the two cases, and it does not correspond to the evident difference in the number of visible lines shown by the data (Fig. 1). Together with the fact that both the calculated temperatures shown in the plots are not high enough to produce a thermal ionization of the K-shell, this is a first indication that the number of $K_{\alpha}$ lines observed in the spectra doesn't depend on the plasma conditions, but rather depends on how many lines have a K-edge energy which is below the FEL photon energy (or for which a resonant transition exists whose final state can radiatively decay through a $\mathrm{K}_{\alpha}$ transition).

Second, the behaviour of the system in the two cases is very different early and late in time: in the first few femtoseconds a faster build-up and subsequent depletion of the $4^{+}$population (recall that $3^{+}$is simply the ground state of the metallic Al sample) is observed in the 1580 $\mathrm{eV}$ case, accompanied by a faster increase in temperature than in the $1830 \mathrm{eV}$ case, whilst the situation is dramatically inverted at later times. This is easily explained by considering that according to these calculations $1830 \mathrm{eV}$ is above the $\mathrm{K}$-edge for all of the possible lines, whilst $1580 \mathrm{eV}$ is above the edge for lines IV and $\mathrm{V}$ only (referring to the standard nomenclature, labelling the emission lines in Fig. 2): for these two lines, corresponding to the photoionization of $3^{+}$and $4^{+}$ions, the ionization cross-section is larger for the lowest energy of $1580 \mathrm{eV}$, so that the energy deposition process is initially more efficient than in the $1830 \mathrm{eV}$ case. However, as soon as the system is ionized up to the $5^{+}$state the only photoabsorption process available at $1580 \mathrm{eV}$ is Lshell ionization, which has a much lower cross-section; conversely, the $1830 \mathrm{eV}$ photons can be absorbed by the $\mathrm{K}$-shell of all of the charge states, so that the energy de- 

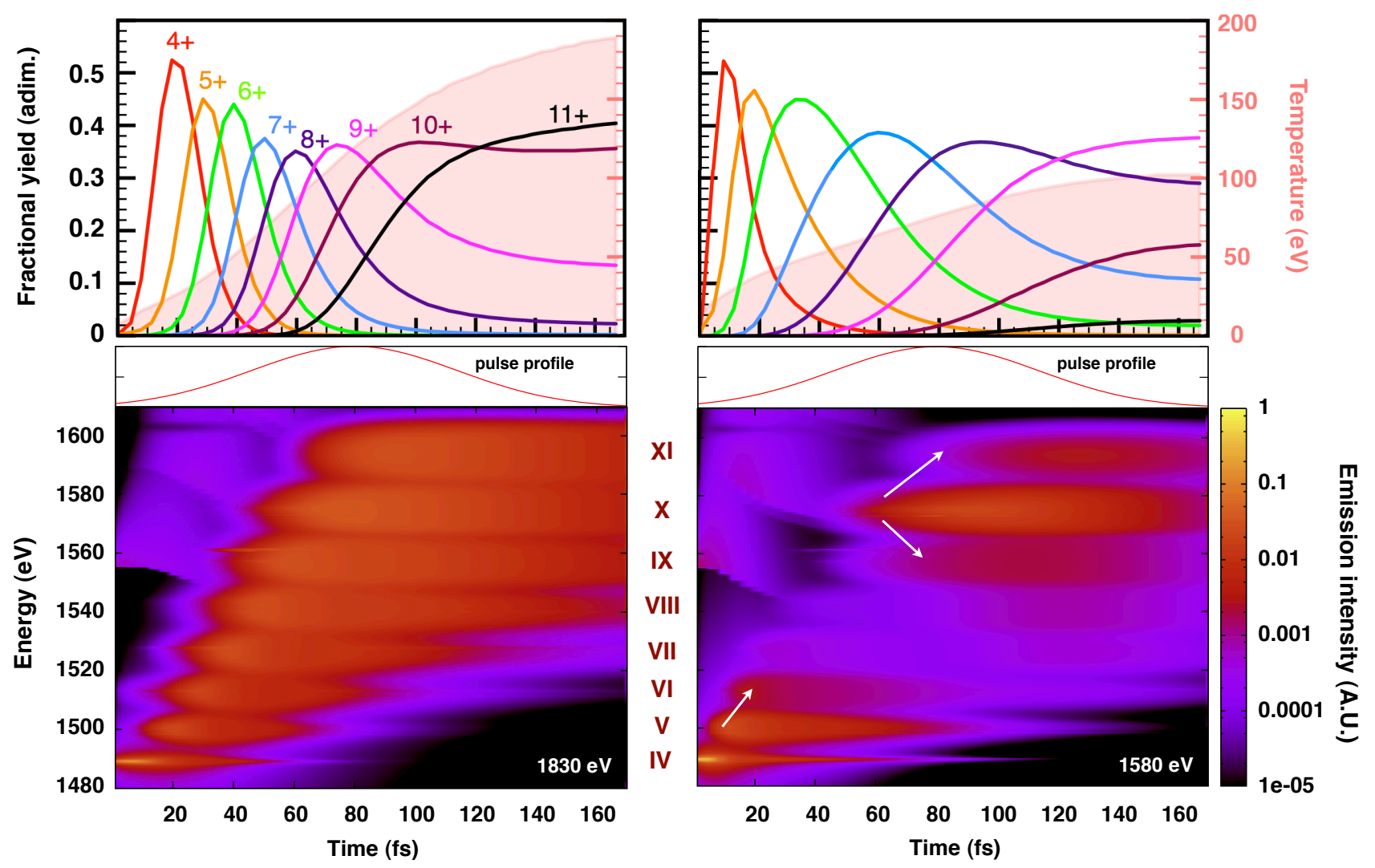

FIG. 2. (Color online) Temporal evolution of the temperature (top, shaded area), charge state distribution (top, lines), and emission spectra (bottom) according to the simulations at the FEL focus. The system is either driven by $1830 \mathrm{eV}$ photons (left plots) or by $1580 \mathrm{eV}$ photons (right plots). The roman numerals denote the standard nomenclature for the corresponding emission lines on the plots. The continuum emission visible at earlier times above $1550 \mathrm{eV}$ in the bottom plots corresponds to the $\mathrm{K}_{\beta}$ series (i.e. free-bound transitions in the case of Aluminum).

position mechanism for charge states higher than $5^{+}$is more efficient for this case, resulting in a greater final temperature and faster ionization chain. It has been recently shown experimentally that this process drives a wavelength-dependent saturable absorption mechanism in the Al sample ${ }^{32}$.

Importantly, although the argument above proves that the K-shell photoionization is the primary energy deposition mechanism, the CSDs shown in Fig. 2 do not imply that the ionization chain itself is driven by the photoionization processes: in particular the CSDs do not show the typical odd-even alternation caused by the Auger decays in photoionization-driven plasmas ${ }^{27-29}$, providing strong evidence for the collisional ionization being the driving process instead. This argument becomes very convincing when comparing how the temperature relates to the CSD in two plots: for any instantaneous value of the temperature the corresponding populations in the two cases are essentially identical. Indeed, performing an LTE calculation with no FEL at few selected temperatures yields the same instantaneous CSD, although the populations of the states with a K-shell hole can be underestimated by several orders of magnitude, given that these particular states are exclusively populated by photoionization.
In other words, the system is found to be in partial LTE, with the populations of the ground states (largely dominant in the CSD) following the Saha equilibrium, but with the states generating the K-alpha spectra entirely dominated by the radiation field.

The resulting picture so far is that of a plasma isochorically heated by the short FEL pulse to temperatures up to $200 \mathrm{eV}$, where most of the charge states are populated at some point during the evolution, but only a subset of them can emit $\mathrm{K}_{\alpha}$ radiation, depending on the FEL photon energy. This picture is quite evidently represented in the time-dependent emission spectra in Fig. 2. When pumping at $1830 \mathrm{eV}$ the laser can photoionize any of the charge states currently available at any point in time, and as a result the emission closely follows the evolution of the CSD. Instead, when pumping at $1580 \mathrm{eV}$ the only lines that significantly emit are line IV and V, for which the FEL is above the K-edge threshold, and which appear when the $3^{+}$and $4^{+}$charge states are populated, and line X, for which the laser can resonantly excite a K-shell electron to the L-shell, and reveals the time at which the $10^{+}$charge state becomes available in the CSD plotted above.

The time-dependent spectrum at $1580 \mathrm{eV}$ also shows 
another interesting effect: the neighbouring lines to the lines that can be photoexcited, namely line $\mathrm{V}$ and line $\mathrm{X}$, also exhibit a lower intensity but still visible emission, which is due to collisional ionization/recombination of L-shell electrons in ions which already have a K-shell hole ${ }^{1,9}$, a process represented in the figure by the white arrows. Note that this collisional propagation of the lines makes the succession for the (low-level) emission of the lines in the $1580 \mathrm{eV}$ case very similar to the succession observed in the $1830 \mathrm{eV}$ one, due to the collisional evolution of the CSD that underlies the ionization chain probed by the FEL in that case. The collisional effect is particularly spectacular in the simulation for line VII, which, although weak, can be produced at early times $(\sim 20 \mathrm{fs})$ by a double L-shell ionization from the initial states of the transition corresponding to line $\mathrm{V}$, and then at later times ( $120 \mathrm{fs})$ by a triple recombination from the resonantly excited states responsible for the emission of line X: the line thus exhibits a double structure in time. These collisional ionization effects predicted by the simulations are observed in the experiment (Fig. 1) and have been recently used to obtain an experimental estimate of collisional ionization rates in hot dense matter ${ }^{9}$.

\section{B. Spatial gradients}

By comparing the spectra in Fig. 1 and Fig. 2 it is clear that it is not possible to reproduce the experimental results by using the instantaneous emission spectrum at any single time step: this would correspond to the often-used plasma diagnostic approach of attempting to associate a single density and temperature with an experimental spectrum. Moreover, although the local simulations provide a fully self-consistent story, which explains the many effects observed in the experiment, it is also clear that a simple time integration of the spectra in Fig. 2 would not work either: this is shown for the $1830 \mathrm{eV}$ case in Fig. 3, where the blue curve is the result of such integration. It is worth noting that if a standard Gaussian profile is approximated by a square profile with the same FWHM, the peak intensity is very similar to the average intensity, a further proof that using average conditions to describe the system doesn't lead to a good description of the spectra.

As mentioned in the introduction, in order to reproduce the experimental spectra we accounted for the spatial profile of the laser, measured during the experiment using an imprints technique ${ }^{25}$. The measurement provides a fluence scan (F-scan) for the laser as a function $F(S)$, where $F$ is the local value of the normalized fluence at any specific point of the focal plane, and $S$ is the surface enclosed by an iso-fluence curve on that plane. In other words, the inverse function of the F-scan, or $S(F)$, describes what portion $S$ of the surface of the target experiences a laser fluence larger than $F$ (where $F=1$ corresponds to peak fluence). Note that the Rayleigh range of the laser has been measured to be of the order

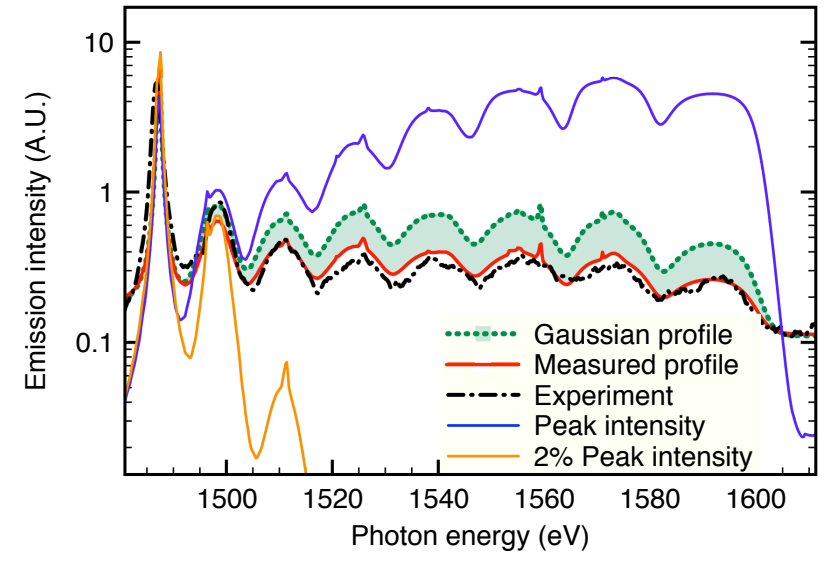

FIG. 3. (Color online) Effect of the laser spatial gradients on the Al spectra, when pumped at $1830 \mathrm{eV}$. The experiment is compared with two local simulations (corresponding to two different peak intensities for the laser pulse) and with two space-integrated simulations, one taking into account the measured spatial profile, and one assuming a Gaussian profile with the same fluence.

of a centimeter ${ }^{33}$, four orders of magnitude larger than the thickness of the target, so that the beam is collimated in the longitudinal direction. Moreover, radiation transport calculations ${ }^{16}$ show a negligible difference $(\sim 5 \%)$ between the final temperatures at the front and at back of the target, so that the system can be approximated as uniform in the longitudinal direction. By inverting $F(S)$, we can thus obtain the volume of the target which is experiencing a particular fluence, and translate that fluence into a local laser intensity history.

Each of the space-time integrated simulations in Fig. 1 is obtained by performing and weighting 26 zerodimensional simulations, spanning five orders of magnitude in peak intensity, each using an intensity profile given by

$$
I_{n}(\omega, t)=I_{0}(\omega, t) e^{-2(n \cdot 0.1)^{2}}
$$

where $I_{0}$ is the focal intensity profile, which has a Gaussian envelope both in time $(t)$ and frequency $(\omega)$ and $n=0, \ldots, 25$. If $I_{-1}$ is defined to be equal to $I_{0}, h$ is the thickness of the target, and $S(I)$ is the inverse function of the F-scan, $F(S)$, expressed in term of intensity rather than fluence, then the weighting factor for each of the simulations is given by

$$
W\left(I_{n}\right)=h \times\left(S\left(\frac{I_{n}+I_{n+1}}{2 I_{0}}\right)-S\left(\frac{I_{n}+I_{n-1}}{2 I_{0}}\right)\right),
$$

which corresponds to dividing the volume of the target into 26 regions of volume $W\left(I_{n}\right)$, according to the measured spatial profile.

The 26 simulations could of course be weighted by assuming an ideal Gaussian beam profile with the same fluence: the result of such an operation is compared with the full simulation using the experimental F-scan in Fig. 3, 


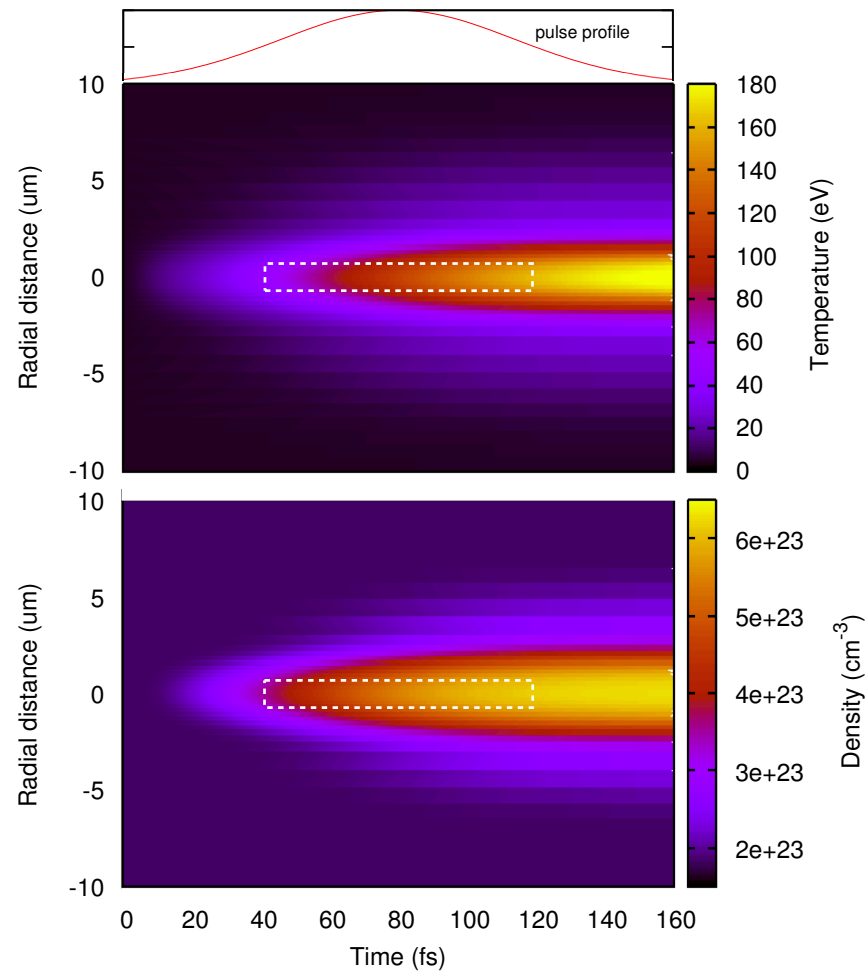

FIG. 4. (Color online) Spatially-resolved temperature and electron density evolution of the plasma driven by $1830 \mathrm{eV}$ photons. The plots assume cylindrical symmetry of the laser pulse, and the rectangular sections are defined by the FWHM of the laser pulse, both in time and in space domain. For reference, an electron density of $6 \times 10^{23} \mathrm{~cm}^{-3}$ corresponds to an average ionization of 10 for the solid density $\mathrm{Al}$ plasma.

for the simulations at an FEL photon energy of 1830 $\mathrm{eV}$. The figure also shows the corresponding experimental spectrum, and the local time-integrated spectra corresponding to the intensities $I_{0}$ and $I_{9}$. As already clear from Fig. 1, the simulation using the measured profile does an excellent job in reproducing the ratio between all of the satellites, whilst it is clear that even a renormalization of the spectrum obtained using the Gaussian profile would be unable to reproduce at the same time the lowand high-energy part of the spectrum, demonstrating the need to accurately measure the intensity distribution on target in order to faithfully reproduce the experimental spectra. The differences between the two cases are due to the fact that according to the experimental F-scan the lower intensities impinge on a much larger part of the volume of the target than in the Gaussian case, which increases the ratio between the lines emitted mainly in the colder parts of the samples (the cold $\mathrm{K}_{\alpha}$ and nearest satellites) and those that can only be emitted in the hotter, highly ionized zones $\left(\mathrm{He}_{\alpha}\right.$ and nearest satellites). The contribution of the different intensities on the different parts of the spectrum is exemplified by the two single-intensity simulations.

If one assumes that the surfaces $S$ defining the $F(S)$ are disks, a cylindrically symmetric analogue of the space-resolved plasma evolution can be built (where space-resolved refers to the transverse direction to the laser, as there are no longitudinal gradients). This is shown in Fig. 4 for the temperature and electron density evolution of the plasma pumped at $1830 \mathrm{eV}$. The figure also shows the region of the space-time plane included within the FWHM of the spatial/temporal laser profile. Although it is clear from the figure that within this specific spatial region the plasma is heated and highly ionized to very uniform conditions shortly after the peak of the pulse, proving the ability of FELs of generating uniform samples of hot-dense matter, it is still important to keep in mind that a conspicuous part of the laser energy is deposited in the low intensity wings: as a result a much larger portion of the target than that within the FWHM is moderately heated and ionized, thus generating the balance between higher and lower charge states shown in Fig. 3.

Although the role of the spatial and temporal gradients is strongly influential in determining the form of the $\mathrm{Al}$ spectra, these gradients do not tend to hinder extracting information about the pertinent physics in well characterized conditions. In fact - as described in section II A - the FEL acts as a probe capable of revealing by spectroscopy a subset of charge states (depending on the wavelength) at the time when their corresponding ground state exists within the plasma, within a duration and set of spatial regions which is restricted to a specific range of temperatures (Fig. 2) and densities by the close-to-LTE condition. An example of how this leads to a well characterized measurement of collisional ionization rates for specific charge states is discussed in the relevant paper ${ }^{9}$.

\section{Auger heating and instantaneous thermalization}

The reliability of the SCFLY simulations discussed so far depends on a good description of the mechanism by which the laser energy is transferred to the plasma, and then transformed into temperature of the free electrons. In SCFLY the self-consistent calculation of the temperature relies on the assumption of instantaneous thermalization of the electron gas. Unfortunately, verifying the validity of such an assumption by standard estimates of the electron-electron collision rate $^{34}$ is non-trivial in strongly coupled conditions ${ }^{35}$, where the Coulomb logarithm is ill-defined. On the other hand, the very high collisional ionization/recombination rates ${ }^{1,9}$ ensure the existence of an efficient mechanism by which the electrons can exchange kinetic energy, through the interaction with the ionic system, on a femtosecond timescale.

Nevertheless, it is clear from the above discussion that the main laser absorption mechanism is K-shell photoabsorption, which will mainly transform the absorbed energy into kinetic energy of Auger electrons, via the KLL Auger decays following the photoionization events (the fluorescence yield for $\mathrm{Al}$ is $\sim 3 \%$ ). One could then easily argue that this would create a marked two temperature 
distribution of the free electrons, where the collisionally ionised electrons, the directly excited photoelectrons, and the original free electrons of the metal have a different characteristic temperature than the Auger electrons. As a result, if this energy stored in the Auger population is not returned to the plasma via collisions as though it were directly stored into a thermal distribution, this could strongly affect the validity and accuracy of the simulations.

In order to investigate the impact of these non-thermal effects on the simulations, the SCFLY model was modified in such a way as to separate the Auger distribution from the thermal distribution. The main idea is to extend the ion population array $N_{i}(t)$, by adding a bin $N_{k}(t)$ for each Auger transition generating a free electron of energy $E_{k}$ : these bins are populated by Auger processes, and depopulated by collisional processes involving electrons at the given energy, with the colliding electron being then transferred to the thermal electron distribution. If $R \delta_{j(k)}$ is the Auger rate for the transition generating an Auger electron of energy $E_{k}$ from the decay of specific state $N_{j}$, and if $R_{i j}^{k}$ is a collisional rate for a transition $N_{j} \rightarrow N_{i}$ involving an Auger electron at $E_{k}$, whilst $R_{i j}$ are the standard rates for the non-LTE model, and $R_{f r}^{k}$ is the Auger-thermal electron collision rate, then the modified rate equations assume the form

$$
\begin{aligned}
\frac{d}{d t}\left(\begin{array}{c}
N_{i} \\
N_{k}
\end{array}\right)= & \sum_{j \neq i}\left(\begin{array}{c}
R_{i j}+\sum_{k} R_{i j}^{k} N_{k} \\
R \delta_{j(k)}-\sum_{i} R_{i j}^{k} N_{k}
\end{array}\right) N_{j}+ \\
& -\left(\begin{array}{c}
\sum_{j \neq i}\left[R_{j i}+\sum_{k} R_{j i}^{k} N_{k}\right] N_{i} \\
R_{f r}^{k} n_{e} N_{k}
\end{array}\right) .
\end{aligned}
$$

The rates $R_{i j}^{k}$ are calculated using the standard SCFLY routines for non-thermal electrons ${ }^{36}$, assuming a monochromatic distribution for each bin, whilst $R_{f r}^{k}$ is estimated ${ }^{34}$ by using the Coulomb logarithm defined by Lee et $a l .{ }^{37}$. The energy balance is correspondingly modified to take into account what portion of the absorbed energy is retained by the Auger distribution, and what portion is transferred back to the thermal part of the plasma by collisions.

The results of a simulation driven at $1630 \mathrm{eV}$ performed with the modified model is shown in Fig. 5. The plot shows a perfect overlap between the density and temperature history predicted by the modified model and those calculated by a standard SCFLY simulation. Correspondingly, the population of the Auger bins is shown never to exceed $0.2 \%$ of the total number of free electrons. This excellent agreement is easily explained by considering the energy transfer rates plotted in the bottom part of the figure: whenever part of the energy absorbed by the ions from the laser (Laser $\rightarrow$ Plasma) is transformed into kinetic energy of the Auger electrons via Auger decays (Plasma $\rightarrow$ Auger), that energy is immediately returned to either the ions or the thermal electrons by collisional processes (Auger $\rightarrow$ Plasma), thus preventing a substantial build-up of an Auger population. Note that even if

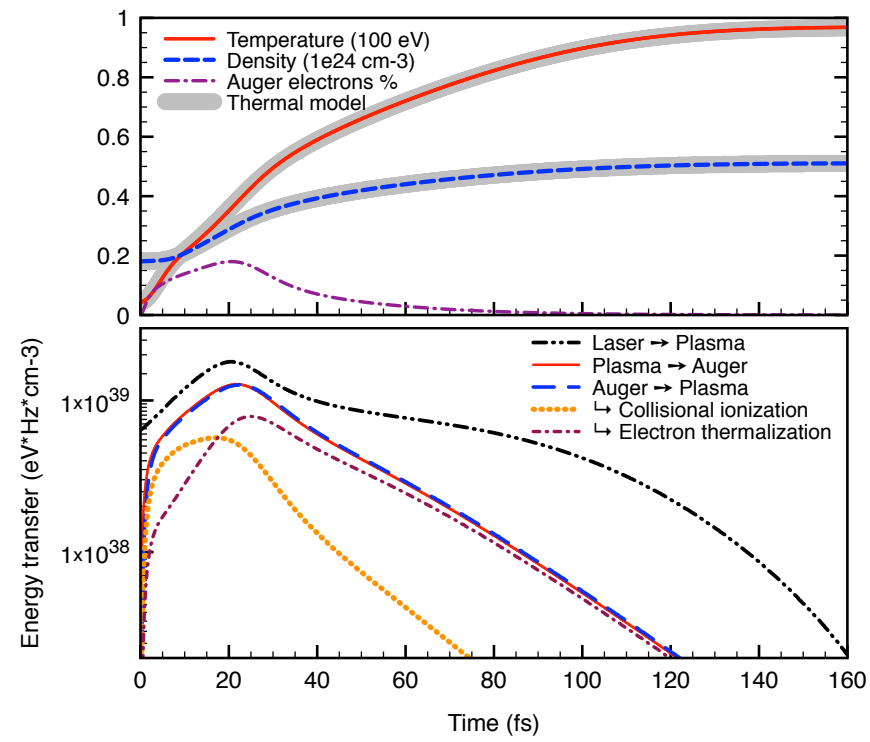

FIG. 5. (Color online) Plasma evolution (top) and energy transfer (bottom) for the modified SCFLY model including an Auger distribution, in the case of a single-intensity simulation at $1630 \mathrm{eV}$. The temperature and density evolution (units in legend) are compared with those given by a standard simulation (Thermal model). The energy transfer from the Auger bins to the plasma is decomposed in its main components due to Auger-ion collisions, and Auger-thermal electrons collisions.

in practice it takes a few collisions in order for an Auger electron to fully thermalize, the mechanism preventing the formation of non-thermal components of the electron distribution is still well captured by this simplified model.

The divergence between the laser absorption profile and the energy stored in the Auger bins, shown in Fig. 5 at later times, is due to the fact that at these times the plasma is ionized beyond the maximum charge state that can still absorb the laser photons by photoionizing the Kshell: at this point only L-shell photoionization (with a lower absorption rate) is possible, which doesn't lead to the generation of further Auger electrons. However the photoelectrons generated by this process have kinetic energies which are very similar to the Auger energies, and could be treated in the same fashion, credibly yielding similar results.

Finally, the figure also shows the two main contributions to the energy transfer from the Auger system to the plasma, namely the contribution of Auger-ion collisional ionization (a comparison of the Auger collisional rates with the thermal and photoionization rates for the same simulation in Fig. 5 is also shown in the recent paper on collisional rates $^{9}$ ), and the contribution of the collisions between Auger and thermal electrons. Although this last contribution dominates the energy transfer in this simulation at certain times, note that the aforementioned intrinsic uncertainties introduced by the estimate of $R_{f r}^{k}$ do not actually affect the final results: a simulation performed by setting $R_{f r}^{k}=0$ (the worst case scenario of 

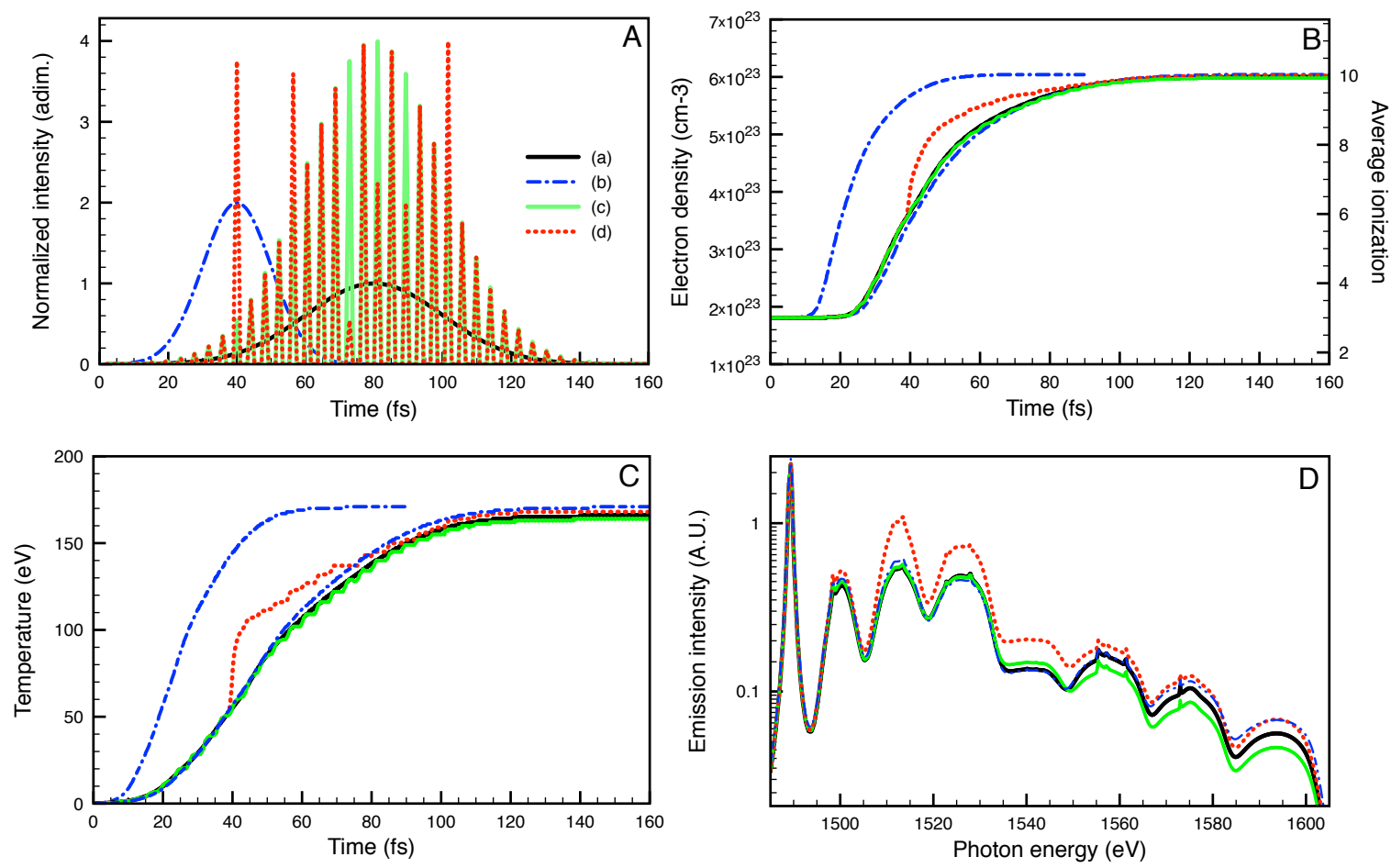

FIG. 6. (Color online) Local simulations at an FEL photon energy of $1650 \mathrm{eV}$ using different laser pulse profiles. A - Temporal profiles. The legend is common to the four plots. All of the pulses have the same fluence. B,C - Evolution of the electron densities and temperatures for the four simulations. The curves for simulation (b) are plotted both in their standard form $f(t)$ and in the form $f(t / 2)$, which allows a direct comparison with the other cases. D - Time-integrated spectra for the four simulations.

no electron-electron thermalization) yields exactly the same energy transfer curves, but with the Auger-ion term completely dominating the transfer. The very high collisionality of the system, either due to the ions or to the thermal electrons, still prevents the build-up of nonthermal components of the electron distribution, ensuring the validity of the instantaneous thermalization assumption. The higher-than-predicted collisional rates estimated from the experiment ${ }^{9}$, would only result in an even faster thermalization process.

Recently, a Fokker-Planck treatment of non-thermal electrons was implemented into an atomic kinetics calculation to model more comprehensively the free-electron relaxation dynamics in a neon plasma irradiated by an Xray $\mathrm{FEL}^{38}$. Calculations using the same model, but conducted for an $\mathrm{Al}$ plasma at solid density ${ }^{39}$, show results which are consistent with the overall plasma evolution presented above using our somewhat simpler model.

\section{FEL temporal structure}

Given that the purpose of the SCFLY simulations is to reproduce the data with minimal input from the modeler, the main source of uncertainty in simulating the FEL pulse interacting with the $\mathrm{Al}$ sample is the temporal structure of the pulse itself. The issue is twofold. First, the chaotic structure of the SASE pulse is characterized by random intensity spikes, which might be thought to potentially affect the reproducibility of spectral features that are strongly intensity dependent, even for data which are averaged over several shots ${ }^{27}$. Although we mentioned that this explains the poorer agrement of the simulations with the double-core-hole lines shown in Fig. 1, its impact on the plasma evolution (and its diagnostic given by the main $\mathrm{K}_{\alpha}$ series) deserves further investigation. Second, the pulse duration of the LCLS is poorly known, and its nominal value is given by the duration of the electron bunch generating the X-ray pulse, which represents just an upper limit for the real pulse length. This value has been used in all of the SCFLY simulations, but several independent investigations ${ }^{28,29,40,41}$ provide strong evidence that the real pulse duration is actually much shorter.

In order to investigate the impact of these uncertainties on the modelling, we performed a set of simulations with different laser profiles. These profiles are shown in Fig. 6, together with the resulting temperature and density histories, and the corresponding emission spectra. The four simulations are $I_{0}$ simulations at an FEL photon energy of $1650 \mathrm{eV}$, and they all have the same pulse fluence; compared are an 80 fs Gaussian pulse (a), a 40 fs Gaussian profile (b), a pulse composed of 1 fs (FWHM) triangular spikes modulated by the 80 fs Gaussian (c), and a 
pulse built from the latter by shuffling a few spikes (d), which roughly mimics the random amplitude of the SASE spikes, still preserving the same fluence and duration as the pulse (c). The pulse duration is here defined as a $1 / e^{2}$ duration, which gives slightly shorter pulses than those defined in the previous simulations (this is closer to the actual pulse duration, and also enhances possible perturbations due to the short-duration spikes).

The density and temperature evolution for the four simulations are compared in subfigure $\mathrm{B}$ and $\mathrm{C}$ respectively. For the case of the short pulse (b), the two curves are also plotted by stretching the time axis by a factor of 2 , in order to verify the overlap with the longer pulses. Recalling the fast collisional evolution described in section II A, it is perhaps not surprising that the final densities and temperature are all roughly the same. In the particular case of simulations (a) and (b), the only difference is that the energy deposition occurs twice as fast in the 40 fs case, which just means that this energy is converted into ionization - via collisions - twice as fast, and the overall plasma evolution will look almost exactly the same as in the $80 \mathrm{fs}$ simulation, after an appropriate rescaling of the time axis. The same applies to the remaining simulations (c) and (d), where instantaneous increases in the absorption are balanced out by periods of low absorption, with the result that the plasma evolution is momentarily hastened or slowed, only to reproduce the same final conditions.

However, instantaneous differences in the laser intensity are not entirely without effect, as can be verified in two instances. First, a small divergence between the temperatures of cases (a) and (b) can be observed at a time of $\sim 50 \mathrm{fs}$. By looking at plot B of Fig. 6, this time corresponds to an average ionization of $\sim 7$ for the plasma: $7^{+}$ ions undergoing a K-shell photoionization can then resonantly absorb a second FEL photon $(1650 \mathrm{eV})$ to create a second K-shell hole, leading to the resonant emission observed in Fig. 1. This two-photon process is clearly enhanced by the higher overall intensity of the shorter simulation (b), leading to a slightly larger absorption for this case. Note however that this does not happen for simulation (c), whose spikes at 50 fs have comparable intensity to that of simulation (b) at the relevant time of 25 fs: this happens because the lifetime of a charge state (see Fig. 2) is longer than the duration of the spikes, so that periods of increased absorption cancel out with periods of decreased absorption in simulation (c), which then closely follows simulation (a). The very small difference in temperature, and the negligible effect on the final spectrum for simulation (b) shown in Fig. 6-D, suggest that we can safely ignore this sort of effect.

Second, if one allows the intensity spikes to behave more randomly, as in the case of simulation (d), the argument used above for simulation (c) about the lifetime of the charge states may well break down. At 40 fs, simulation (d) has a sudden increase in intensity by more than an order of magnitude: this not only affects the temperature and density in a much more visible way

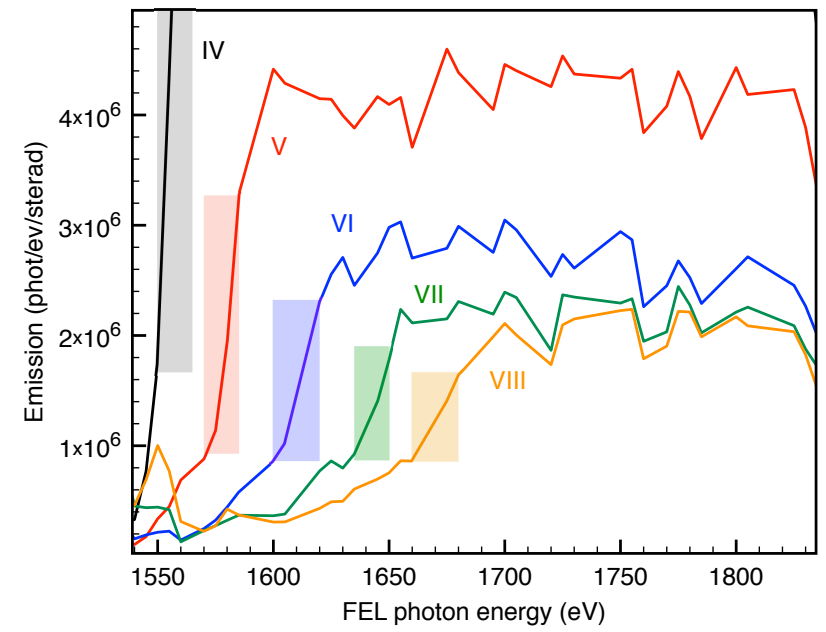

FIG. 7. (Color online) Measured emission thresholds for lines VI-VIII. The curves can be obtained from the color-encoded plot in Fig. 1, by taking vertical lineouts for each of the emission lines. The shaded regions correspond to the K-edge regions as quoted on the continuum lowering paper ${ }^{8}$.

(though still preserving their final values), but it also allows for a much larger number of photons than in any of the other cases to interact with the specific charge states populated at that time, even if this number is integrated over the charge state lifetime. As a result a visible difference for the emission from those particular ion stages is observed in Fig. 6-D. Nevertheless, this effect is still unlikely to be visible in the current experimental results, which are obtained by averaging the spectra obtained in several shots at each FEL photon energy: over a few shots these intensity fluctuations will be randomly distributed over the time window, and as such they are unlikely to enhance particular features in the integrated spectra. Experiments using a shorter pulse length, with duration much closer to that of the spikes, may be able to observe such effects.

The simulations can thus be considered predictive independently from the details of the temporal envelope and from the actual duration of the FEL pulse, provided that the simulated pulse has the right fluence and spatial distribution, corresponding to the ones measured during the experiment. Finally, we stress that even in the worst case scenario of a single simulation (d) with a strong off-peak intensity spike - as observed in Fig. 6-D - the chaoticity of the SASE pulse cannot affect the ratio between lines for which the FEL is above/below the corresponding K-edge: the below-edge emission is indeed purely generated by collisional processes from the neighbouring lines that can be photopumped, as described in section II A. As a result it cannot affect the conclusions for the K-edge measurement work ${ }^{8}$, nor those for the collisional rate measurement work ${ }^{9}$. 


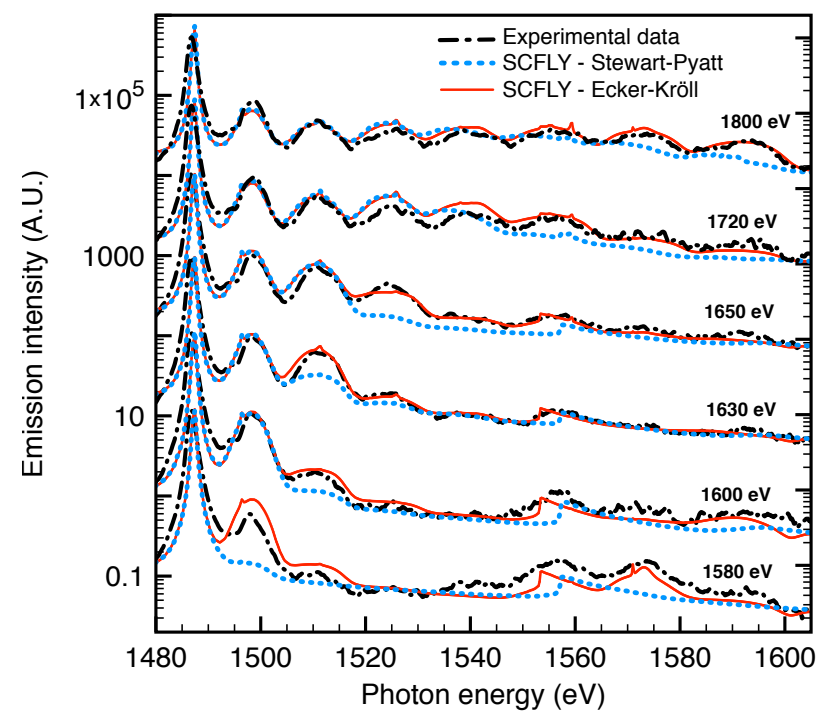

FIG. 8. (Color online) Comparison between the experimental spectra and the prediction of the SCFLY simulations using either the EK, SP model, or fixed K-edges (as determined by the experiment). The chosen FEL photon energies show the onset of the line emission for line VII and VIII, well described by EK and the fixed-edges simulations, but not by the SP model.

\section{CHARGE RESOLVED MEASUREMENTS OF CONTINUUM LOWERING}

Having a model which is able to reproduce the full plethora of effects shown by the experimental spectra with no input from the user regarding the plasma conditions, only of the characteristics of the X-ray pulse provides confidence in the physical parameters employed. In particular, this result is key in enabling us to extract from the data a charge-resolved measurement of K-edge energies, and thus of continuum lowering.

As shown in Fig. 1, the experimental spectra exhibit clear emission lines corresponding to $\mathrm{Al}$ ion configurations with a varying number of L-shell holes. We observe that as the X-ray pump energy is varied, the intensity of these lines changes considerably. In particular, as shown in Fig. 7, we have recorded clear and relatively narrow threshold energies for the emission from ions containing an increasingly vacant L-shell. We stress that while the onset of emission from a specific line could be indicative of a K-shell ionization process, it could also be due to inner-shell excitation. The resonance spectra clearly show such an effect, where bright emission is observed from ions excited at a photon energy in resonance with the K-L bound-bound excitation. As such, an emission threshold alone is not necessarily a measurement of a $\mathrm{K}$ edge energy.

However, an ionization event and an inner-shell excitation event behave differently: they give rise to different charge state distributions, and have different absorption cross sections and emission rates, which all influence the total emission spectrum. One can thus assume different models for the ionization potential depression in the plasma - in each case fully consistent with the evolution of the system dictated by the X-ray absorption and the dynamics governed by the non-LTE rate equations - and investigate which models yield spectra consistent with the experiment and which do not. The result of such a comparison between the SP and EK IPD models is given in Fig. 8 for a range of FEL photon energies. We observe distinctly different predictions from the two models, with the calculations using the EK functional form for the IPD showing close agreement with all the experimental spectra collected, while the calculations using the SP form for the IPD do not match the line intensities for several emission peaks for most FEL photon energies.

The EK IPD model depresses the ionization energies so that no $\mathrm{Al}$ ion has a bound M-shell state. The charge state of an ion in such a plasma is thus determined by the K- and L-shell occupation alone, and therefore within this model the emission lines correspond to well-defined charge states. In this case the threshold energies are thus also ionization energies of the K-shell, i.e., the K-edges. The SP model predicts different IPD energies for all ions, but for the first three holes in the L-shell of Al also does not admit bound M-shell states. Thus, both the SP and EK models predict the first three thresholds to correspond to the K-edges of the system, and can be compared with the experimentally measured edges directly. For higher charge states this is no longer true within the SP model ${ }^{8}$, because with additional bound M-shell states the observed emission thresholds could be due to some combination of K-shell ionization with or without M-shell spectators, or to $\mathrm{K}-\mathrm{M}$ resonant transitions.

The agreement between the data and the simulations using the EK model is seen to be very good across the full range of experimental results. Given how challenging it is to perform predictive spectral modelling in such hot-dense plasmas, this result is somewhat surprising, and it is instructive to investigate whether we can identify an underlying physical reason behind the agreement. For this purpose, we performed a range of simulations with the exact same parameters as above, but substituting the IPD model with fixed IPD energies for each Al charge state, determined to be such that the K-edges of the various ions were consistent with the observed experimental emission thresholds. This corresponds to a very simplistic IPD model, which ignores all of the complex temperature and density effects in the continuum lowering process. Coincidentally, these energies turn out to be sufficiently high to never re-bind the M-shell states. We show the synthetic spectra produced with this model in Fig. 9 for the onset of the emission for line VI and VII, where an excellent agreement with the experimental results and with the simulations using the EK model can be observed. We conclude that the main reason for an IPD model to yield spectra consistent with the experiment within an atomic kinetics simulation - is for the observed emission thresholds to be identified as the K-edges of the 


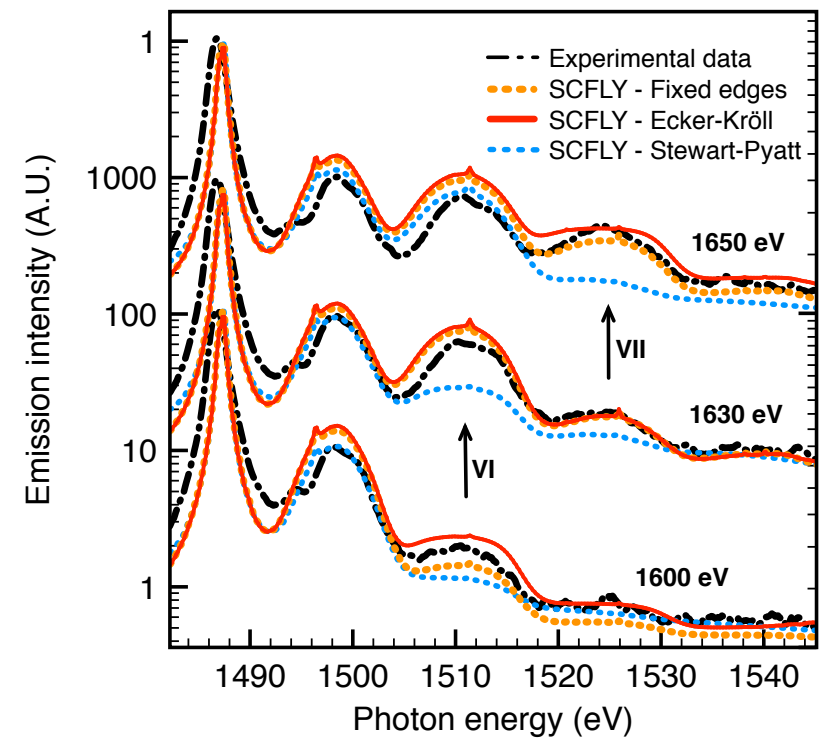

FIG. 9. (Color online) Comparison between the experimental spectra and the prediction of the SCFLY simulations using either the EK, SP model, or fixed K-edges (as determined by the experiment). The chosen FEL photon energies show the onset of the line emission for line VII and VIII, well described by EK and the fixed-edges simulations, but not by the ones using the SP model.

system. We stress that this is not a trivial observation, as there is no a priori reason nor requirement for the thresholds to be K-edges, and indeed in some IPD models (i.e., SP for high charge states) they are not.

It is possible that some set of ad-hoc modifications to various parameters of a specific simulation (e.g. Xray intensity and distribution on target, sample density and temperature, detailed line positions, line broadenings etc.) combined with the SP (or some other) IPD model, could be constructed to produce a simulation to match the corresponding experimental result. Such a fitting process would however involve fine tuning the simulation parameters for each individual experimental condition and would therefore be able to provide little insight into the underlying physics, over and beyond the assumptions used for the fit. Indeed, once a specific model is assumed correct within a fitting process or algorithm, any fitting result will necessarily be consistent with that model. For this reason we have avoided this approach altogether, and the only parameter that is changed between all the different simulations presented is the X-ray photon energy, as determined in the experiment, and the functional form for the IPD.

Finally, it is important to note that the IPD-model approach to simulating continuum lowering within an atomic kinetics simulation is a very simplistic way of treating density effects in a plasma. By necessarily having to define a state as either fully free or fully bound it is especially poorly suited to describing the continuous transition between bound and free states in the insulator-metal transition with increasing density due to electron delocalization and band formation. As such, the description based on IPD models is particularly inappropriate when IPD energies are similar to the energies of atomic valence states, where banding processes would be expected to be significant, and can lead to unphysical effects where small variations in IPD energies yield very different partition functions for the plasma-embedded ion. In such cases extra care is warranted and more advanced simulation techniques would be required for a detailed interpretation of the results.

For the above reason, an alternative description of the system, based on a Density Functional Theory (DFT) approach - a description that does not rely on pressure ionization - has been recently developed ${ }^{42}$. The density of states predicted by the DFT model shows a temperaturedependent structure for the valence band, and exhibits the onset of relocalization effects occurring in the proximity of a continuum threshold, which are not captured by any analytical continuum lowering model. However, the position of the threshold levels and the lack of clearly identifiable fully localized states for the M-shell are in excellent agreement with the interpretation of the data provided by the SCFLY simulations using the EK model for aluminum.

\section{CONCLUSIONS}

We have described the details of the simulation work concerning the experiment presented in Vinko et al. ${ }^{1}$, where the X-ray pulse of the LCLS isochorically heats a thin $\mathrm{Al}$ foil to the hot dense regime, and the $\mathrm{K}_{\alpha}$ emission from the sample is used to diagnose the plasma.

Even though the simulations show that a proper treatment of the spatial and temporal gradients is crucial in order to reproduce the experimental spectra, local and instantaneous properties can explain many features observed in the data. Whilst an accurate description ${ }^{25}$ of the spatial profile of the laser is required in order to describe the spectra (which are poorly described by a simple Gaussian profile), the requirements on the knowledge of the exact temporal profile or even the pulse duration of the FEL are much less stringent, and a good knowledge of the pulse fluence is enough to reproduce the data. We have shown that effects due to the chaotic nature of the SASE pulse of the LCLS may become relevant only for data obtained in single shot mode, in agreement with previous independent investigations ${ }^{27}$.

The SCFLY simulations described here are not fits to the data, but predictions based solely on the FEL parameters. This is achieved by exploiting the simple laser energy deposition mechanism granted by the X-ray wavelengths of the LCLS: the single-photon absorption events can be easily tracked, and are intrinsically included in a non-LTE treatment of the plasma evolution. Nevertheless, the laser absorption and the temperature evolution of the plasma are related by the assumption of instan- 
taneous thermalization of the electrons, which needs a non-trivial validation for these plasma conditions. We have shown that a modified SCFLY model, that tracks the populations of the fast Auger electrons produced in the interaction, confirms that the high collisionality of the system suppresses the growth of a non-thermal component of the electron distribution, so that the instantaneous thermalization approximation provides a valid description of the Al plasma.

Having obtained predictive simulations, which are not biased by any user choice about the density of the system, we have explained how this leads to a benchmark comparison of different continuum lowering models ${ }^{8}$, completely independent of any a priori assumption about the charge state of the emitting ions, that can be influenced by pressure ionization effects. The excellent description of the data in terms of the EK model, leads to a one-to-one correspondence between the observed emission lines and the charge states of the emitting ions, thus turning the benchmark experiment into a direct charge-resolved measurement of continuum lowering.

Nevertheless, we have discussed that relocalization effects, which could not be described by any continuum lowering approach, may still occur, as captured by a more recent DFT approach ${ }^{42}$ : this does not mean that the most appropriate continuum lowering description (approximate in nature) may not still be the one which treats such semi-localized states as free.

Finally, we note that such a discrepancy with SP, firstly pointed out by Ciricosta et $a l^{8}{ }^{8}$, is not to be resolved at any cost: apart from the weak coupling nature of that model, which makes it mathematically unsuitable for the LCLS experimental conditions, a higherthan-predicted IPD has also been later observed experimentally at Orion $^{10}$, and at Omega ${ }^{43}$, and several independent theoretical models have been recently developed claiming better agreement than SP with both the LCLS and Orion data ${ }^{12,13,44}$. Moreover, the more physical description of the system provided by the DFT calculations $^{42}$, not reliant on any pressure ionization effect, is in excellent agreement with the approximate picture provided by the SCFLY simulations using the EK model: although we do not expect that model to work in a large range of conditions of element, density and temperature (as stressed in the continuum lowering paper ${ }^{8}$ ), its description of the system - in the context of the purely predictive simulation approach described in this work appears reliable.

\section{ACKNOWLEDGEMENTS}

Use of the Linac Coherent Light Source (LCLS), SLAC National Accelerator Laboratory, is supported by the U.S. Department of Energy, Office of Science, Office of Basic Energy Sciences under Contract No. DE-AC0276SF00515". The SXR Instrument is funded by a consortium whose membership includes the LCLS, Stanford
University through the Stanford Institute for Materials Energy Sciences (SIMES), Lawrence Berkeley National Laboratory (LBNL), University of Hamburg through the BMBF priority program FSP 301, and the Center for Free Electron Laser Science (CFEL). O.C., S.M.V., D.S.R. and J.S.W. thank the UK EPSRC for funding (EP/F020449/1, EP/H035877/1, and EP/L000849/1). S.M.V. gratefully acknowledges support from the Royal Society.

${ }^{1}$ S. M. Vinko, O. Ciricosta, B. I. Cho, K. Engelhorn, H.-K. Chung, C. R. D. Brown, T. Burian, J. Chalupský, R. W. Falcone, C. Graves, V. Hjkov, A. Higginbotham, L. Juha, J. Krzywinski, H. J. Lee, M. Messerschmidt, C. D. Murphy, Y. Ping, A. Scherz, W. Schlotter, S. Toleikis, J. J. Turner, L. Vysin, T. Wang, B. Wu, U. Zastrau, D. Zhu, R. W. Lee, P. A. Heimann, B. Nagler, and J. S. Wark, Nature (London) 482, 59 (2012).

${ }^{2}$ B. I. Cho, K. Engelhorn, S. M. Vinko, H.-K. Chung, O. Ciricosta, D. S. Rackstraw, R. W. Falcone, C. R. D. Brown, T. Burian, J. Chalupský, C. Graves, V. Hájková, A. Higginbotham, L. Juha, J. Krzywinski, H. J. Lee, M. Messersmidt, C. Murphy, Y. Ping, N. Rohringer, A. Scherz, W. Schlotter, S. Toleikis, J. J. Turner, L. Vysin, T. Wang, B. Wu, U. Zastrau, D. Zhu, R. W. Lee, B. Nagler, J. S. Wark, and P. A. Heimann, Physical Review Letters 109, 245003 (2012).

${ }^{3}$ T. Guillot, $\quad$ Science $286, \quad 72 \quad$ (1999), http://www.sciencemag.org/content/286/5437/72.full.pdf.

${ }^{4}$ D. B. Guenther, P. Demarque, Y.-C. Kim, and M. H. Pinsonneault, The Astrophysical Journal 387, 372 (1992).

${ }^{5}$ C. A. Iglesias and F. J. Rogers, The Astrophysical Journal 371 , 408 (1991).

${ }^{6}$ J. D. Lindl, P. Amendt, R. L. Berger, S. G. Glendinning, S. H. Glenzer, S. W. Haan, R. L. Kauffman, O. L. Landen, and L. J. Suter, Physics of Plasmas 11, 339 (2004).

${ }^{7}$ S. X. Hu, B. Militzer, V. N. Goncharov, and S. Skupsky, Phys. Rev. Lett. 104, 235003 (2010)

${ }^{8}$ O. Ciricosta, S. M. Vinko, H.-K. Chung, B.-I. Cho, C. R. D. Brown, T. Burian, J. Chalupský, K. Engelhorn, R. W. Falcone, C. Graves, V. Hájková, A. Higginbotham, L. Juha, J. Krzywinski, H. J. Lee, M. Messerschmidt, C. D. Murphy, Y. Ping, D. S. Rackstraw, A. Scherz, W. Schlotter, S. Toleikis, J. J. Turner, L. Vysin, T. Wang, B. Wu, U. Zastrau, D. Zhu, R. W. Lee, P. Heimann, B. Nagler, and J. S. Wark, Phys. Rev. Lett. 109, 065002 (2012).

${ }^{9}$ S. Vinko, O. Ciricosta, T. R. Preston, D. S. Rackstraw, C. Brown, T. Burian, J. Chalupský, B. I. Cho, H.-K. Chung, K. Engelhorn, R. W. Falcone, R. Fiokovinini, V. Hjkov, P. A. Heimann, L. Juha, H. J. Lee, R. W. Lee, M. Messerschmidt, B. Nagler, W. Schlotter, J. J. Turner, L. Vysin, U. Zastrau, and J. S. Wark, Nature Communications 6, 6397 (2015).

${ }^{10}$ D. J. Hoarty, P. Allan, S. F. James, C. R. D. Brown, L. M. R. Hobbs, M. P. Hill, J. W. O. Harris, J. Morton, M. G. Brookes, R. Shepherd, J. Dunn, H. Chen, E. Von Marley, P. Beiersdorfer, H. K. Chung, R. W. Lee, G. Brown, and J. Emig, Physical Review Letters 110, 265003 (2013).

${ }^{11}$ L. B. Fletcher, A. L. Kritcher, A. Pak, T. Ma, T. Döppner, C. Fortmann, L. Divol, O. S. Jones, O. L. Landen, H. A. Scott, J. Vorberger, D. A. Chapman, D. O. Gericke, B. A. Mattern, G. T. Seidler, G. Gregori, R. W. Falcone, and S. H. Glenzer, Phys. Rev. Lett. 112, 145004 (2014).

${ }^{12}$ S.-K. Son, R. Thiele, Z. Jurek, B. Ziaja, and R. Santra, Phys. Rev. X 4, 031004 (2014).

${ }^{13}$ B. Crowley, High Energy Density Physics 13, 84 (2014).

${ }^{14}$ C. A. Iglesias, High Energy Density Physics 12, 5 (2014).

${ }^{15}$ S. B. Hansen, J. Colgan, A. Y. Faenov, J. Abdallah, S. A. Pikuz, I. Y. Skobelev, E. Wagenaars, N. Booth, O. Culfa, R. J. Dance, G. J. Tallents, R. G. Evans, R. J. Gray, T. Kaempfer, K. L. Lancaster, P. McKenna, A. K. Rossall, K. S. Schulze, I. Uschmann, 
A. G. Zhidkov, and N. C. Woolsey, Physics of Plasmas 21, 031213 (2014).

${ }^{16}$ D. Rackstraw, S. Vinko, O. Ciricosta, B. Cho, K. Engelhorn, H.-K. Chung, C. Brown, T. Burian, J. Chalupsk, R. Falcone, C. Graves, V. Hjkov, A. Higginbotham, L. Juha, J. Krzywinski, H. Lee, M. Messerschmidt, C. Murphy, Y. Ping, A. Scherz, W. Schlotter, S. Toleikis, J. Turner, L. Vysin, T. Wang, B. Wu, U. Zastrau, D. Zhu, B. Nagler, R. Lee, P. Heimann, and J. Wark, High Energy Density Physics 11, 59 (2014).

${ }^{17}$ J. C. Stewart and K. D. Pyatt Jr., Astrophysical Journal 144, 1203 (1966)

${ }^{18}$ G. Ecker and W. Kröll, Physics of Fluids 6, 62 (1963).

${ }^{19}$ T. R. Preston, S. M. Vinko, O. Ciricosta, H.-K. Chung, R. W. Lee, and J. S. Wark, High Energy Density Physics 9, 258 (2013).

${ }^{20}$ H.-K. Chung, M. Chen, W. Morgan, Y. Ralchenko, and R. Lee, High Energy Density Phys. 1, 3 (2005).

${ }^{21}$ H.-K. Chung, M. Chen, and R. Lee, High Energy Density Phys. 3, 57 (2007).

${ }^{22}$ A. Bar-Shalom, J. Oreg, W. H. Goldstein, D. Shvarts, and A. Zigler, Phys. Rev. A 40, 3183 (1989).

${ }^{23}$ M. H. Chen, B. Crasemann, and H. Mark, Phys. Rev. A 21, 436 (1980).

${ }^{24}$ J. Apruzese, Journal of Quantitative Spectroscopy and Radiative Transfer 34, 447 (1985).

${ }^{25}$ J. Chalupský, J. Krzywinski, L. Juha, V. Hájková, J. Cihelka, T. Burian, L. Vysín, J. Gaudin, A. Gleeson, M. Jurek, A. Khorsand, D. Klinger, H. Wabnitz, R. Sobierajski, M. Störmer, K. Tiedtke, and S. Toleikis, Optics Express 18, 27836 (2010).

${ }^{26}$ R. Bonifacio, L. De Salvo, P. Pierini, N. Piovella, and C. Pellegrini, Phys. Rev. Lett. 73, 70 (1994).

${ }^{27}$ N. Rohringer and R. Santra, Physical Review A 76, 033416 (2007).

${ }^{28}$ L. Young, E. P. Kanter, B. Krässig, Y. Li, A. M. March, S. T. Pratt, R. Santra, S. H. Southworth, N. Rohringer, L. F. DiMauro, G. Doumy, C. A. Roedig, N. Berrah, L. Fang, M. Hoener, P. H. Bucksbaum, J. P. Cryan, S. Ghimire, J. M. Glownia, D. A. Reis, J. D. Bozek, C. Bostedt, and M. Messerschmidt, Nature 466, 56 (2010).

${ }^{29}$ O. Ciricosta, H.-K. Chung, R. W. Lee, and J. S. Wark, High Energy Density Phys. 7, 111 (2011).

${ }^{30}$ A. A. Sorokin, U. Jastrow, P. Juranić, S. Kapitzki, K. Tiedtke, M. Richter, U. Arp, S. Moeller, J. Turner, and W. Schlotter, "Report on Pulse energy monitoring at the SXR beamline using gas-monitor detectors," (Technical report, SLAC, 2010).

${ }^{31}$ H. R. Griem, "Plasma Spectroscopy," (McGraw-Hill, 1964).

${ }^{32}$ D. S. Rackstraw, O. Ciricosta, S. M. Vinko, B. Barbrel, T. Burian, J. Chalupský, B. I. Cho, H.-K. Chung, G. L. Dakovski, K. Engelhorn, V. Hájková, P. Heimann, M. Holmes, L. Juha, J. Krzywinski, R. W. Lee, S. Toleikis, J. J. Turner, U. Zastrau, and J. S. Wark, Phys. Rev. Lett. 114, 015003 (2015).

${ }^{33}$ J. Chalupský, P. Bohacek, V. Hájková, S. Hau-Riege, P. Heimann, L. Juha, J. Krzywinski, M. Messerschmidt, S. Moeller, B. Nagler, M. Rowen, W. Schlotter, M. Swiggers, and J. Turner, Nuclear Instruments and Methods in Physics Research Section A: Accelerators, Spectrometers, Detectors and Associated Equipment 631, 130 (2011).

${ }^{34}$ J. D. Huba, "NRL Plasma Formulary," Naval Research Laboratory, Washington, D.C. (2009).

${ }^{35}$ D. O. Gericke, M. S. Murillo, and M. Schlanges, Physical Review E 65, 036418 (2002).

${ }^{36}$ H.-K. Chung, R. Lee, M. Chen, and Y. Ralchenko, "The How To for FLYCHK (NIST,2008),".

${ }^{37}$ Y. T. Lee and R. M. More, Physics of Fluids 27, 1273 (1984).

${ }^{38}$ A. de la Varga, P. Velarde, F. de Gaufridy, D. Portillo, M. Cotelo, A. Barbas, A. González, and P. Zeitoun, High Energy Density Physics 9, 542 (2013).

${ }^{39} \mathrm{P}$. Velarde (private communication, 2015).

${ }^{40}$ J. Wu, Y. Ding, P. Emma, Z. Huang, H. Loos, M. Messerschmidt, E. Schneidmiller, and M. Yurkov, in Proceedings of the 2010 Free-Electron Laser Conference.
${ }^{41}$ S. Düsterer, P. Radcliffe, C. Bostedt, J. Bozek, A. L. Cavalieri, R. Coffee, J. T. Costello, D. Cubaynes, L. F. DiMauro, Y. Ding, G. Doumy, F. Grüner, W. Helml, W. Schweinberger, R. Kienberger, A. R. Maier, M. Messerschmidt, V. Richardson, C. Roedig, T. Tschentscher, and M. Meyer, New Journal of Physics 13, 093024 (2011).

${ }^{42} \mathrm{~S}$. Vinko, O. Ciricosta, and J. Wark, Nature Communications 5, 3533 (2014).

${ }^{43}$ L. B. Fletcher, A. Kritcher, A. Pak, T. Ma, T. Dppner, C. Fortmann, L. Divol, O. L. Landen, J. Vorberger, D. A. Chapman, D. O. Gericke, R. W. Falcone, and S. H. Glenzer, Physics of Plasmas (1994-present) 20, 056316 (2013).

${ }^{44}$ A. Calisti, S. Ferri, and B. Talin, Contributions to Plasma Physics 55, 360 (2015). 